\title{
Article
}

\section{On some new subclass of bi-univalent functions associated with the Opoola differential operator}

\section{Timilehin Gideon Shaba}

Department of Mathematics, University of Ilorin, P. M. B. 1515, Ilorin, Nigeria.; shabatimilehin@gmail.com

Received: 21 June 2020; Accepted: 25 August 2020; Published: 31 August 2020.

Abstract: By applying Opoola differential operator, in this article, two new subclasses $\mathcal{M}_{\mathcal{H}, \sigma}^{\mu, \beta}(m, \psi, k, \tau)$ and $\mathcal{M}_{\mathcal{H}, \sigma}^{\mu, \beta}(m, \xi, k, \tau)$ of bi-univalent functions class $\mathcal{H}$ defined in $\nabla$ are introduced and investigated. The estimates on the coefficients $\left|l_{2}\right|$ and $\left|l_{3}\right|$ for functions of the classes are also obtained.

Keywords: Univalent function, bi-univalent function, coefficient bounds, Opoola differential operator.

MSC: 30C45, 30C50.

\section{Introduction}

$\mathbf{L}$

et $\mathcal{J}$ denote the subclass of $\mathcal{G}$ which is of the form

$$
\Im(z)=z+\sum_{k=2}^{\infty} l_{k} z^{k}
$$

consisting of functionas which are holomorphic and univalent in the unit disk $\nabla$. Let $\Im^{-1}$ be inverse of the function $\Im(z)$, then we have

$$
\Im^{-1}(\Im(z))=z
$$

and

where

$$
\Im^{-1}(\Im(b))=b, \quad|b|<r_{0}(\Im) ; r_{0}(\Im) \geq \frac{1}{4}
$$

$$
\Im^{-1}(\Im(b))=b-l_{2} b^{2}+\left(2 l_{2}^{2}-l_{3}\right) b^{3}-\left(5 l_{2}^{3}-5 l_{2} l_{3}+l_{4}\right) b^{4}+\cdots
$$

A function $\Im(z) \in \mathcal{G}$ denoted by $\mathcal{H}$ is said to be bi-univalent in $\nabla$ if both $\Im(z)$ and $\Im^{-1}(z)$ are univalent in $\triangle$ [1]. Subclasses of $\mathcal{H}$, such as class of bi-convex and starlike functions and bi-strongly convex and starlike function similar to the well known subclasses $\mathcal{L}^{*}(\vartheta)$ and $\mathcal{K}(\vartheta)$ of starlike and convex functions of order $\vartheta(0<$ $\vartheta<1)$ respectively [2].

Recently, numerous researchers [1,3,4] obtained the coefficient $\left|l_{2}\right|$ and $\left|l_{3}\right|$ of bi-univalent functions for the several subclasses of functions in the class $\mathcal{H}$. Motivated by the work of Darus and Singh [5], we introduce the subclasses $\mathcal{M}_{\mathcal{H}}^{\mu, \beta}(m, \psi, k, \tau)$ and $\mathcal{M}_{\mathcal{H}, \sigma}^{\mu, \beta}(m, \xi, k, \tau)$ of the function class $\mathcal{H}$, which are associated with the Opoola differential operator and to obtain estimates on the coefficients $\left|l_{2}\right|$ and $\left|l_{3}\right|$ for functions in these new subclasses of the function class $\mathcal{H}$ applying the techniques used earlier by Darus and Singh [5], Frasin and Aouf [4] and Srivastava et al., [1].

Lemma 1. [6] Suppose $u(z) \in \mathcal{P}$ and $z \in \nabla$, then $\left|w_{k}\right| \leq 2$ for each $k$, where $\mathcal{P}$ is the family of all function $u$ analytic in $\nabla$ for which $\Re(u(z))>0$,

$$
u(z)=1+w_{1} z+w_{2} z^{2}+\cdots .
$$


Definition 1. A function $\Im(z) \in \mathcal{G}$ is in the class $\mathcal{M}_{\mathcal{H}, \sigma}^{\mu, \beta}(m, \psi, \tau)$ if the following condition are fulfilled:

$$
\begin{gathered}
\left|\arg \left[\frac{(1-\sigma) D_{\tau, \beta}^{m, \mu} \Im(z)+\sigma D_{\tau, \beta}^{m+1, \mu} \Im(z)}{z}\right]\right|<\frac{\psi \pi}{2}, \\
\left|\arg \left[\frac{(1-\sigma) D_{\tau, \beta}^{m, \mu} h(b)+\sigma D_{\tau, \beta}^{m+1, \mu} h(b)}{b}\right]\right|<\frac{\psi \pi}{2},
\end{gathered}
$$

where $0<\psi \leq 1, \sigma \geq 1, \tau \geq 0, z \in \triangle, b \in \triangle, 0 \leq \mu \leq \beta, m \in \mathcal{N}_{0}$ and

$$
h(b)=b-l_{2} b^{2}+\left(2 l_{2}^{2}-l_{3}\right) b^{3}-\left(5 l_{2}^{3}-5 l_{2} l_{3}+l_{4}\right) b^{4}+\cdots
$$

and

$$
D_{\tau, \beta}^{m, \mu} \Im(z)=z+\sum_{k=2}^{\infty}(1+(k+\mu-\beta-1) \tau)^{m} l_{k} z^{k}
$$

where $\quad 0 \leq \mu \leq \beta, \tau \geq 0$ and $m \in \mathbb{N}_{0}=\{0,1,2,3 \cdots\}$ is the generalized Al-oboudi derivative defined by Opoola [7].

\section{Remark 1. .}

1. $\mathcal{M}_{\mathcal{H}, 1}^{\mu, \beta}(0, \psi, \tau)=\mathcal{M}_{\mathcal{H}}(\psi)$ which Srivastava et al., [1] presented and studied.

2. $\mathcal{M}_{\mathcal{H}, \sigma}^{\mu, \beta}(0, \psi, \tau)=\mathcal{M}_{\mathcal{H}, \sigma}(\psi)$ which Frasin and Aouf [4] presented and studied.

3. $\mathcal{M}_{\mathcal{H}, \sigma}^{1,1}(m, \psi, 1)=\mathcal{M}_{\mathcal{H}, \sigma}(m, \psi)$ which Porwal and Darus [8] presented and studied.

4. $\mathcal{M}_{\mathcal{H}, \sigma}^{1,1}(m, \psi, \tau)=\mathcal{M}_{\mathcal{H}, \sigma}(m, \psi, \tau)$ which Darus and Singh [5] presented and studied.

2. Coefficient Bounds For The Function Class $\mathcal{M}_{\mathcal{H}}^{\mu, \beta}(m, \psi, k, \tau)$

Theorem 1. Let $\Im(z) \in \mathcal{G}$ be in the class $\mathcal{M}_{\mathcal{H}}^{\mu, \beta}(m, \psi, k, \tau), 0<\psi \leq 1, \sigma \geq 1, \tau \geq 0, z \in \triangle, b \in \triangle, 0 \leq \mu \leq \beta$, $m \in \mathcal{N}_{0}$, then

$$
\left|l_{2}\right| \leq \frac{2 \psi}{\sqrt{\begin{array}{c}
2 \psi\left[(1-\sigma)(1+\tau(2+\mu-\beta))^{m}+\sigma(1+\tau(2+\mu-\beta))^{m+1}\right]- \\
\psi(\psi-1)\left[(1-\sigma)(1+\tau(1+\mu-\beta))^{m}+\sigma(1+\tau(1+\mu-\beta))^{m+1}\right]^{2}
\end{array}}}
$$

and

$$
\begin{aligned}
\left|l_{3}\right| \leq & \frac{2 \psi}{\left[(1-\sigma)(1+\tau(2+\mu-\beta))^{m}+\sigma(1+\tau(2+\mu-\beta))^{m+1}\right]} \\
& +\frac{4 \psi^{2}}{\left[(1-\sigma)(1+\tau(2+\mu-\beta))^{m}+\sigma(1+\tau(2+\mu-\beta))^{m+1}\right]}
\end{aligned}
$$

Proof. It follows from (3) and (4) that

$$
\frac{(1-\sigma) D_{\tau, \beta}^{m, \mu} \Im(z)+\sigma D_{\tau, \beta}^{m+1, \mu} \Im(z)}{z}=(q(z))^{\psi},
$$

and

$$
\frac{(1-\sigma) D_{\tau, \beta}^{m, \mu} h(b)+\sigma D_{\tau, \beta}^{m+1, \mu} h(b)}{b}=(t(b))^{\psi},
$$

where $q(z)=1+q_{1} z+q_{2} z^{2}+q_{3} z^{3}+\cdots$ and $t(b)=1+t_{1} b+t_{2} b^{2}+t_{3} b^{3} \cdots$ are in $\mathcal{P}$. Equating the coefficient in (9) and (10), we have

$$
\left[(1-\sigma)(1+\tau(1+\mu-\beta))^{m}+\sigma(1+\tau(1+\mu-\beta))^{m+1}\right] l_{2}=\psi q_{1}
$$




$$
\begin{gathered}
{\left[(1-\sigma)(1+\tau(2+\mu-\beta))^{m}+\sigma(1+\tau(2+\mu-\beta))^{m+1}\right] l_{3}=\psi q_{2}+\frac{\psi(\psi-1)}{2} q_{1}^{2},} \\
-\left[(1-\sigma)(1+\tau(1+\mu-\beta))^{m}+\sigma(1+\tau(1+\mu-\beta))^{m+1}\right] l_{2}=\psi t_{1}, \\
{\left[(1-\sigma)(1+\tau(2+\mu-\beta))^{m}+\sigma(1+\tau(2+\mu-\beta))^{m+1}\right]\left(2 l_{2}^{2}-l_{3}\right)=\psi t_{2}+\frac{\psi(\psi-1)}{2} t_{1}^{2} .}
\end{gathered}
$$

From (11) and (13), we get

$$
q_{1}=-t_{1}
$$

and

$$
2\left[(1-\sigma)(1+\tau(1+\mu-\beta))^{m}+\sigma(1+\tau(1+\mu-\beta))^{m+1}\right]^{2} l_{2}^{2}=\psi^{2}\left(q_{1}^{2}+t_{1}^{2}\right) .
$$

From (12),(14) and (16), we get

$$
\begin{aligned}
& 2\left[(1-\sigma)(1+\tau(1+\mu-\beta))^{m}+\sigma(1+\tau(1+\mu-\beta))^{m+1}\right]^{2} l_{2}^{2} \\
& -\left[(1-\sigma)(1+\tau(1+\mu-\beta))^{m}+\sigma(1+\tau(1+\mu-\beta))^{m+1}\right]^{2} l_{3}=\psi t_{2}+\frac{\psi(\psi-1)}{2} t_{1}^{2}
\end{aligned}
$$

implies

$$
\begin{aligned}
& 2\left[(1-\sigma)(1+\tau(1+\mu-\beta))^{m}+\sigma(1+\tau(1+\mu-\beta))^{m+1}\right]^{2} l_{2}^{2} \\
& =\left[(1-\sigma)(1+\tau(1+\mu-\beta))^{m}+\sigma(1+\tau(1+\mu-\beta))^{m+1}\right]^{2} l_{3}+\psi t_{2}+\frac{\psi(\psi-1)}{2} t_{1}^{2} .
\end{aligned}
$$

Then from (12), we have

$$
2\left[(1-\sigma)(1+\tau(1+\mu-\beta))^{m}+\sigma(1+\tau(1+\mu-\beta))^{m+1}\right]^{2} l_{2}^{2}=\psi q_{2}+\frac{\psi(\psi-1)}{2} q_{1}^{2}+\psi t_{2}+\frac{\psi(\psi-1)}{2} t_{1}^{2},
$$

implies

$$
2\left[(1-\sigma)(1+\tau(1+\mu-\beta))^{m}+\sigma(1+\tau(1+\mu-\beta))^{m+1}\right]^{2} l_{2}^{2}=\psi\left(q_{2}+t_{2}\right)+\frac{\psi(\psi-1)}{2}\left(q_{1}^{2}+t_{1}^{2}\right) .
$$

Then from (16), we get

$$
\begin{aligned}
& 2\left[(1-\sigma)(1+\tau(1+\mu-\beta))^{m}+\sigma(1+\tau(1+\mu-\beta))^{m+1}\right]^{2} l_{2}^{2} \\
& =\psi\left(q_{2}+t_{2}\right)+\frac{\psi(\psi-1)}{2} \frac{2\left[(1-\sigma)(1+\tau(1+\mu-\beta))^{m}+\sigma(1+\tau(1+\mu-\beta))^{m+1}\right]^{2}}{\psi^{2}} l_{2}^{2},
\end{aligned}
$$

implies

$$
l_{2}^{2}=\frac{\psi^{2}\left(q_{2}+t_{2}\right)}{2 \psi\left[(1-\sigma)(1+\tau(2+\mu-\beta))^{m}+\sigma(1+\tau(2+\mu-\beta))^{m+1}\right]-} .
$$

Applying Lemma 1 for (17), we get

$$
\left|l_{2}\right| \leq \frac{2 \psi}{\sqrt{\begin{array}{c}
2 \psi\left[(1-\sigma)(1+\tau(2+\mu-\beta))^{m}+\sigma(1+\tau(2+\mu-\beta))^{m+1}\right]- \\
\psi(\psi-1)\left[(1-\sigma)(1+\tau(1+\mu-\beta))^{m}+\sigma(1+\tau(1+\mu-\beta))^{m+1}\right]^{2}
\end{array}}}
$$

which gives the desired estimate on $\left|l_{2}\right|$ in (7). Hence in order to find the bound on $\left|l_{3}\right|$,

$$
\begin{aligned}
& {\left[(1-\sigma)(1+\tau(2+\mu-\beta))^{m}+\sigma(1+\tau(2+\mu-\beta))^{m+1}\right] l_{3}-\left[(1-\sigma)(1+\tau(2+\mu-\beta))^{m}\right.} \\
& \left.+\sigma(1+\tau(2+\mu-\beta))^{m+1}\right]\left(2 l_{2}^{2}-l_{3}\right)=\psi q_{2}+\frac{\psi(\psi-1)}{2} q_{1}^{2}-\left[\psi t_{2}+\frac{\psi(\psi-1)}{2} t_{1}^{2}\right],
\end{aligned}
$$


implies

$$
\begin{aligned}
& 2\left[(1-\sigma)(1+\tau(2+\mu-\beta))^{m}+\sigma(1+\tau(2+\mu-\beta))^{m+1}\right] l_{3} \\
& =\left[(1-\sigma)(1+\tau(2+\mu-\beta))^{m}+\sigma(1+\tau(2+\mu-\beta))^{m+1}\right] 2 l_{2}^{2}+\psi\left(q_{2}-t_{2}\right)+\frac{\psi(\psi-1)}{2}\left(q_{1}^{2}-t_{1}^{2}\right) .
\end{aligned}
$$

Since $\left(q_{1}\right)^{2}=\left(-t_{1}\right)^{2} \Longrightarrow q_{1}^{2}=t_{1}^{2}$, then we have

$$
\begin{aligned}
& 2\left[(1-\sigma)(1+\tau(2+\mu-\beta))^{m}+\sigma(1+\tau(2+\mu-\beta))^{m+1}\right] l_{3} \\
& =\psi\left(q_{2}-t_{2}\right)+\left[(1-\sigma)(1+\tau(2+\mu-\beta))^{m}+\sigma(1+\tau(2+\mu-\beta))^{m+1}\right] 2 l_{2}^{2} \\
& l_{3}=\frac{\psi\left(q_{2}-t_{2}\right)}{2\left[(1-\sigma)(1+\tau(2+\mu-\beta))^{m}+\sigma(1+\tau(2+\mu-\beta))^{m+1}\right]} \\
& \quad+\frac{\left[(1-\sigma)(1+\tau(2+\mu-\beta))^{m}+\sigma(1+\tau(2+\mu-\beta))^{m+1}\right]}{2\left[(1-\sigma)(1+\tau(2+\mu-\beta))^{m}+\sigma(1+\tau(2+\mu-\beta))^{m+1}\right]} 2 l_{2}^{2} .
\end{aligned}
$$

From (16), we have

$$
\begin{aligned}
l_{3}= & \frac{\psi\left(q_{2}-t_{2}\right)}{2\left[(1-\sigma)(1+\tau(2+\mu-\beta))^{m}+\sigma(1+\tau(2+\mu-\beta))^{m+1}\right]} \\
& +\frac{\psi^{2}\left(q_{1}^{2}+t_{1}^{2}\right)}{2\left[(1-\sigma)(1+\tau(1+\mu-\beta))^{m}+\sigma(1+\tau(1+\mu-\beta))^{m+1}\right]^{2}} .
\end{aligned}
$$

Applying Lemma 1 for coefficient $q_{1}, q_{2}, t_{1}$ and $t_{2}$, we have

$$
\begin{aligned}
\left|l_{3}\right| \leq & \frac{2 \psi}{\left[(1-\sigma)(1+\tau(2+\mu-\beta))^{m}+\sigma(1+\tau(2+\mu-\beta))^{m+1}\right]} \\
& +\frac{4 \psi^{2}}{\left[(1-\sigma)(1+\tau(1+\mu-\beta))^{m}+\sigma(1+\tau(1+\mu-\beta))^{m+1}\right]^{2}} .
\end{aligned}
$$

\section{Coefficient bounds for the function class $\mathcal{M}_{\mathcal{H}, \sigma}^{\mu, \beta}(m, \xi, k, \tau)$}

Definition 2. A function $\Im(z) \in \mathcal{G}$ is said to be in the class $\mathcal{M}_{\mathcal{H}, \sigma}^{\mu, \beta}(m, \xi, k, \tau)$ if the following condition are fulfilled:

$$
\begin{aligned}
& \Re\left[\frac{(1-\sigma) D_{\tau, \beta}^{m, \mu} \Im(z)+\sigma D_{\tau, \beta}^{m+1, \mu} \Im(z)}{z}\right]>\xi, \\
& \Re\left[\frac{(1-\sigma) D_{\tau, \beta}^{m, \mu} h(b)+\sigma D_{\tau, \beta}^{m+1, \mu} h(b)}{b}\right]>\xi,
\end{aligned}
$$

where $\Im(z) \in \mathcal{H}, 0 \leq \xi<1, \sigma \geq 1, \tau \geq 0, z \in \triangle, b \in \triangle, 0 \leq \mu \leq \beta, m \in \mathcal{N}_{0}$, and

$$
h(b)=b-l_{2} b^{2}+\left(2 l_{2}^{2}-l_{3}\right) b^{3}-\left(5 l_{2}^{3}-5 l_{2} l_{3}+l_{4}\right) b^{4}+\cdots,
$$

and

$$
D_{\tau, \beta}^{m, \mu} \Im(z)=z+\sum_{k=2}^{\infty}(1+(k+\mu-\beta-1) \tau)^{m} l_{k} z^{k},
$$

where $\quad 0 \leq \mu \leq \beta, \tau \geq 0$ and $m \in \mathbb{N}_{0}=\{0,1,2,3 \cdots\}$ is the generalized Al-oboudi derivative defined by Opoola [7].

\section{Remark 2. .}

1. $\mathcal{M}_{\mathcal{H}, 1}^{\mu, \beta}(0, \xi, \tau)=\mathcal{M}_{\mathcal{H}}(\xi)$ which Srivastava et al., [1] presented and studied. 
2. $\mathcal{M}_{\mathcal{H}, \sigma}^{\mu, \beta}(0, \xi, \tau)=\mathcal{M}_{\mathcal{H}, \sigma}(\xi)$ which Frasin and Aouf [4] presented and studied.

3. $\mathcal{M}_{\mathcal{H}, \sigma}^{1,1}(m, \xi, 1)=\mathcal{M}_{\mathcal{H}, \sigma}(m, \xi)$ which Porwal and Darus [8] presented and studied.

4. $\mathcal{M}_{\mathcal{H}, \sigma}^{1,1}(m, \xi, \tau)=\mathcal{M}_{\mathcal{H}, \sigma}(m, \xi, \tau)$ which Darus and Singh [5] presented and studied.

Theorem 2. Let $\Im(z) \in \mathcal{G}$ be in the class $\mathcal{M}_{\mathcal{H}}^{\mu, \beta}(m, \xi, k, \tau), 0 \leq \xi<1, \sigma \geq 1, \tau \geq 0, z \in \triangle, b \in \triangle, 0 \leq \mu \leq \beta$, $m \in \mathcal{N}_{0}$, then

$$
\left|l_{2}\right| \leq \sqrt{\frac{2(1-\xi)}{\left[(1-\sigma)(1+\tau(2+\mu-\beta))^{m}+\sigma(1+\tau(2+\mu-\beta))^{m+1}\right]}},
$$

and

$$
\begin{aligned}
\left|l_{3}\right| \leq & \frac{4(1-\xi)^{2}}{\left[(1-\sigma)(1+\tau(2+\mu-\beta))^{m}+\sigma(1+\tau(2+\mu-\beta))^{m+1}\right]^{2}} \\
& +\frac{2(1-\xi)}{\left[(1-\sigma)(1+\tau(2+\mu-\beta))^{m}+\sigma(1+\tau(2+\mu-\beta))^{m+1}\right]}
\end{aligned}
$$

Proof. From (18) and (19), where $q(z), t(z) \in \mathcal{P}$,

$$
\frac{(1-\sigma) D_{\tau, \beta}^{m, \mu} \Im(z)+\sigma D_{\tau, \beta}^{m+1, \mu} \Im(z)}{z}=\xi+(1-\xi) q(z),
$$

and

$$
\frac{(1-\sigma) D_{\tau, \beta}^{m, \mu} h(b)+\sigma D_{\tau, \beta}^{m+1, \mu} h(b)}{b}=\xi+(1-\xi) t(b),
$$

where $q(z)=1+q_{1} z+q_{2} z^{2}+q_{3} z^{3}+\cdots$ and $t(b)=1+t_{1} b+t_{2} b^{2}+t_{3} b^{3} \cdots$. Now on equating the coefficient in (24) and (25), we have

$$
\begin{gathered}
{\left[(1-\sigma)(1+\tau(1+\mu-\beta))^{m}+\sigma(1+\tau(1+\mu-\beta))^{m+1}\right] l_{2}=(1-\xi) q_{1},} \\
{\left[(1-\sigma)(1+\tau(2+\mu-\beta))^{m}+\sigma(1+\tau(2+\mu-\beta))^{m+1}\right] l_{3}=(1-\xi) q_{2},} \\
-\left[(1-\sigma)(1+\tau(1+\mu-\beta))^{m}+\sigma(1+\tau(1+\mu-\beta))^{m+1}\right] l_{2}=(1-\xi) t_{1}, \\
{\left[(1-\sigma)(1+\tau(2+\mu-\beta))^{m}+\sigma(1+\tau(2+\mu-\beta))^{m+1}\right]\left(2 l_{2}^{2}-l_{3}\right)=(1-\xi) t_{2} .}
\end{gathered}
$$

From (26) and (28), we have

$$
q_{1}=-t_{1}
$$

and

$$
2\left[(1-\sigma)(1+\tau(1+\mu-\beta))^{m}+\sigma(1+\tau(1+\mu-\beta))^{m+1}\right]^{2} l_{2}^{2}=(1-\xi)^{2}\left(q_{1}^{2}+t_{1}^{2}\right) .
$$

From (27) and (29), we have

$$
2\left[(1-\sigma)(1+\tau(1+\mu-\beta))^{m}+\sigma(1+\tau(1+\mu-\beta))^{m+1}\right] l_{2}^{2}=(1-\xi)\left(q_{2}+t_{2}\right),
$$

or we have

$$
l_{2}^{2}=\frac{(1-\xi)\left(q_{2}+t_{2}\right)}{2\left[(1-\sigma)(1+\tau(1+\mu-\beta))^{m}+\sigma(1+\tau(1+\mu-\beta))^{m+1}\right]}
$$

implies

$$
\left|l_{2}^{2}\right| \leq \frac{2(1-\xi)}{\left[(1-\sigma)(1+\tau(1+\mu-\beta))^{m}+\sigma(1+\tau(1+\mu-\beta))^{m+1}\right]}
$$

which is the bound on $\left|l_{2}\right|$ as given in (22). Hence in order to find the bound on $\left|l_{3}\right|$, we subtract (27) and (29) and get

$$
\begin{aligned}
& {\left[(1-\sigma)(1+\tau(2+\mu-\beta))^{m}+\sigma(1+\tau(2+\mu-\beta))^{m+1}\right] l_{3}} \\
& -\left[(1-\sigma)(1+\tau(2+\mu-\beta))^{m}+\sigma(1+\tau(2+\mu-\beta))^{m+1}\right]\left(2 l_{2}^{2}-l_{3}\right)=(1-\xi) q_{2}-\left[(1-\xi) t_{2}\right],
\end{aligned}
$$


implies

$$
\begin{aligned}
& 2\left[(1-\sigma)(1+\tau(2+\mu-\beta))^{m}+\sigma(1+\tau(2+\mu-\beta))^{m+1}\right] l_{3} \\
& =\left[(1-\sigma)(1+\tau(2+\mu-\beta))^{m}+\sigma(1+\tau(2+\mu-\beta))^{m+1}\right] 2 l_{2}^{2}+(1-\xi)\left(q_{2}-t_{2}\right),
\end{aligned}
$$

implies

$$
l_{3}=l_{2}^{2}+\frac{(1-\xi)\left(q_{2}-t_{2}\right)}{2\left[(1-\sigma)(1+\tau(2+\mu-\beta))^{m}+\sigma(1+\tau(2+\mu-\beta))^{m+1}\right]} .
$$

Then from (31), we have

$$
\begin{aligned}
l_{3}= & \frac{(1-\xi)^{2}\left(q_{1}^{2}+t_{1}^{2}\right)}{2\left[(1-\sigma)(1+\tau(1+\mu-\beta))^{m}+\sigma(1+\tau(1+\mu-\beta))^{m+1}\right]^{2}} \\
& +\frac{(1-\xi)\left(q_{2}-t_{2}\right)}{2\left[(1-\sigma)(1+\tau(2+\mu-\beta))^{m}+\sigma(1+\tau(2+\mu-\beta))^{m+1}\right]}
\end{aligned}
$$

Applying Lemma 1 for the coefficient $q_{1}, q_{2}, t_{1}$ and $t_{2}$, we get

$$
\begin{aligned}
\left|l_{3}\right| \leq & \frac{4(1-\xi)^{2}}{\left[(1-\sigma)(1+\tau(1+\mu-\beta))^{m}+\sigma(1+\tau(1+\mu-\beta))^{m+1}\right]^{2}} \\
& +\frac{2(1-\xi)}{\left[(1-\sigma)(1+\tau(2+\mu-\beta))^{m}+\sigma(1+\tau(2+\mu-\beta))^{m+1}\right]^{\prime}},
\end{aligned}
$$

which is the bond on $\left|l_{3}\right|$ in (23).

\section{Conclusion}

In this present paper, two new subclasses of bi-univalent functions associated with Opoola differential operator $D_{\tau, \beta}^{m, \mu}$ were introduced and worked on. Furthermore, the coefficient bounds for $\left|l_{2}\right|$ and $\left|l_{3}\right|$ of functions in these classes are obtained.

Conflicts of Interest: "The author declares no conflict of interest."

\section{References}

[1] Srivastava, H. M., Mishra, A. K., \& Gochhayat, P. (2010). Certain subclasses of analytic and bi-univalent functions. Applied mathematics letters, 23(10), 1188-1192.

[2] Brannan, D.A., \& Taha, T. (1986). On some classes of bi-univalent functions. Babes-Bolyai Math, 31(2), 70-77.

[3] Xu, Q.H., \& Gui, Y.C., \& Srivastava, H.M. (2012). A certain general subclass of analytic and bi-univalent functions and associated coefficient estimate problems. Applied Mathematics and Computation, 218, 11461-11465.

[4] Frasin, B.A., \& Aouf, M.K. (2011). New subclasses of bi-univalent functions. Applied Mathematics Letters, 24, 1569-1573.

[5] Darus, M., \& Singh, S. (2018). On some new classes of bi-univalent functions. Journal of Applied Mathematics, Statistics and Informatics, 14, 19-26.

[6] Pommerenke, C.H. (1975). Univalent Functions. Vandendoeck and Rupercht, Gottingen.

[7] Opoola, T.O. (2017). On a subclass of univalent function defined by generalized differential operator. International Journal of Mathematical Analysis, 11, 869-876.

[8] Porwal, S., \& Darus, M. (2013). On a class of bi-univalent functions. Journal of Egyptian Mathematical Society, 21, 190-193. 